significant correlation between overall job satisfaction and managerial roles leader $(\mathrm{r}=0.272, \mathrm{p}=0.002)$, decision maker $(\mathrm{r}=0.196, \mathrm{p}=0.022)$, innovator $(\mathrm{r}=0.206, \mathrm{p}=$ $0.014)$ and negotiator $(\mathrm{r}=0.237, \mathrm{p}=0.005)$. There was no correlation between total job satisfaction and motivation of health care professionals $(r=-0.033 ; p=0.713)$.

Conclusion The finding suggests that the nature of work, supervision and coworkers had a modest effect on job satisfaction of healthcare workers at surgery departments. Strengthening of managerial skills would lead to increased job satisfaction of health workers.

\section{P-193 ASSOCIATION BETWEEN SLEEP-WAKE ACTIVITY CIRCADIAN RHYTHM AND MILD COGNITIVE IMPAIRMENT AMONG WORKERS WITH PNEUMOCONIOSIS IN HONG KONG}

1'Shelly Lap-ah TSE, Chun Yuk Jason Tsang, Bixia Huang, Chi Chiu Leung. ${ }^{1}$ The Chinese University of Hong Kong, Hong Kong

\subsection{6/OEM-2021-EPI.226}

Introduction Weakened circadian activity rhythm was found to increase dementia and mild cognitive impairment (MCI) among female aged general population, but little is known among pneumoconiosis workers with male predominating.

Objectives This study aims to investigate sleep-wake activity circadian rhythm of patients with pneumoconiosis and test the hypothesis that weakened circadian rhythm is associated with MCI.

Methods We randomly enrolled patients with pneumoconiosis who participated in the annual interview activity. Community controls frequency-matched by age and sex were recruited as the reference. Participants wore wrist actigraphs continuously for 7 days $(168$ h) after completion of a standardized epidemiological questionnaire. Mesor, doubled amplitude acrophase and percent rhythm are the main circadian parameters and a higher value indicates a more robustness of circadian rhythm. MCI status was assessed using validated Chinese version of Montreal Cognitive Assessment (HK-MoCA) and Mini-mental state examination (MMSE) with a cutoff of $21 / 22$ and 26/27, respectively. Co-variance analysis and multivariate logistic regression were performed to obtain adjusted mean of cognitive score and adjusted odds ratio (AOR).

Results Workers with pneumoconiosis had significantly lower MoCA score than the community controls (20.5 \pm 0.4 vs. 22.0 $\pm 0.5, p=0.03)$ after age and education were adjusted, while the difference in MMSE score was borderline $(25.6 \pm 0.3$ vs. $26.4 \pm 0.4, p=0.08)$. Mesor and Double amplitude were the circadian parameters that were notably lower among pneumoconiosis workers than the community controls. Compared with community controls with higher Mesor values, pneumoconiosis workers with lower Mesor levels had an AOR of 2.89 (95\%CI: 1.20-6.95) for MCI measured by MoCA, which was relatively higher than that of the community controls (AOR $=2.73$, 95\%CI: 0.94-7.93). Similar but attenuated results were observed for double amplitude.

Conclusion This study provides the first epidemiological evidence that increased risks of mild cognitive impairment is associated with weakened circadian rhythm among pneumoconiosis workers.

\section{P-194 SEX DIFFERENCE OF NEGATIVE EMOTION AND CONTRIBUTING FACTORS AMONG CHINESE NIGHTSHIFT WORKERS}

'Shelly Lap-ah TSE, Feng Wang, Zhimin Li. 'The Chinese University of Hong Kong, Hong Kong

\subsection{6/OEM-2021-EPI.227}

Introduction Nightshift work is prevalent in developed and developing countries in which female nightshift workers are more vulnerable, particularly for mental health.

Objective This study aims to investigate if the prevalence of negative emotion differ among male and female nightshift workers and explore the contributing factors using the baseline information of a prospective nightshift worker cohort in Shenzhen, China.

Methods We recruited 5329 workers and collected their blood/urine samples from 5 industries at the baseline, but only included 834 workers from 2 companies into this report because other 3 companies mainly composed of male workers. We adopted a standardized questionnaire to collect information on lifetime nightshift work, lifestyle factors, housework demands and socio-demographic data.

Results Overall, 510 workers were males (61.2\%) and 324 were females (38.8\%). More female than male workers were nightshift workers $(90.1 \%$ vs. $82.7 \%)$, aged $\geq 40$ years $(20.4 \%$ vs. $12.9 \%)$ and married $(74.4 \%$ vs. $58.2 \%)$ but less females attained college or above $(8.6 \%$ vs. $23.5 \%)$ and leisure-time exercises $(32.7 \%$ vs. $50.0 \%)$. Significantly more female workers did different housework including cooking $(12.7 \%$ vs. 9.6\%), washing $(50.6 \%$ vs. $31.4 \%)$ and taking care of children/elderly $(20.4 \%$ vs. $12.2 \%)$, and the sex difference in housework demands was particularly prominent among nightshift workers. Female nightshift workers were more prone to negative emotion because of 'feel exhaust or insufficient energy $(40.1 \%$ vs. $32.2 \%)$ ', 'worry of significant change of body weight ( $18.5 \%$ vs. $12.6 \%)$ ', 'insomnia/poor sleep $(31.5 \%$ vs. $21.3 \%)$ ', and 'hard to concentration or forgetful $(30.1 \%$ vs. $20.4 \%$ )'; however, there was no significant sex difference of negative emotion among daytime workers.

Conclusion This study reveals that female nightshift workers were more vulnerable to negative emotion, and nightshift work schedule and high housework burden are the contributing factors.

\section{P-195 EFFECTIVENESS OF COVID-19 VACCINE IN HEALTH CARE WORKERS, MILAN, ITALY}

${ }^{1}$ Dario Consonni, Patrizia Bono, Massimo Oggioni, Sara Uceda Renteria, Alessandra Piatti, Silvana Castaldi, Antonio Muscatello, Michele Carugno, Luciano Riboldi, Ferruccio Ceriotti, Alessandra Bandera, Andrea Gori, Angela Cecilia Pesatori, Andrea Lombardi. 'Fondazione IRCCS Ca' Granda Ospedale Maggiore Policlinico, Italy

\subsection{6/OEM-2021-EPI.228}

Introduction Randomized controlled trials showed efficacy of vaccines against coronavirus disease 19 (COVID-19). There is the need to quantify vaccine effectiveness in real-word contexts, including people at high risk of infection with severe acute respiratory syndrome coronavirus 2 (SARS-CoV-2), such as health care workers (HCWs).

Objectives To evaluate vaccine effectiveness among hospital HCWs. 